\title{
La inmunología en Antioquia: una historia con futuro. II. La consolidación de una disciplina
}

\author{
Luis F. García ${ }^{1}$
}

\section{RESUMEN}

La inmunología es una de las ciencias biomédicas con mayor desarrollo en la segunda parte del siglo XX y principios del presente en el mundo, también, ha tenido un desarrollo muy importante en Antioquia a partir de los años sesenta del siğlo pasado. En la segunda parte de este recuento histórico, el autor relata los desarrollos ocurridos a finales de los años setenta, en la década de 1980 y la primera mitad de la década de 1990 que llevaron a la creación de los laboratorios, grupos de investigación y programas de posgrado en inmunología que permitieron que esta sea una de las disciplinas biomédicas más sobresalientes en la Universidad de Antioquia.

\section{PALABRAS CLAVE}

Antioquia; Historia; Inmunología

\section{SUMMARY}

\section{Immunology in Antioquia: a history with future. II. The consolidation of a discipline}

Immunology is one of the biomedical sciences that had, worldwide, a greater development in the last part of the XX century and the beginning of the present century. In Antioquia, immunology also had important developments that began the 1960s. In the second part of this historical review, the author describes the developments that occurred in the last years of the 1970s, the 1980s and the first half of the 1990s that lead to the establishment

\footnotetext{
1 Profesor emérito, Grupo de Inmunología Celular e Inmunogenética, Facultad de Medicina, Sede de Investigación Universitaria, Universidad de Antioquia, Medellín, Colombia.

Correspondencia: Luis F. García; Ifgarcia@une.net.co

Recibido: abril 12 de 2019

Aceptado: julio 4 de 2019
}

Cómo citar: García LF. La inmunología en Antioquia: una historia con futuro. II. La consolidación de una disciplina. latreia. 2020 Abr-Jun;33(2):191-198. D0I 10.17533/udea. iatreia.49. 
of the laboratories, groups and graduated programs Immunology that allowed immunology to be one of the more outstanding biomedical disciplines in the University of Antioquia.

\section{KEY WORDS}

Antioquia; History; Immunology

\section{INTRODUCCIÓN}

En la primera parte de esta reseña presenté una breve descripción de la historia de la inmunología con el fin de entender el contexto en el que se inició la creación de una escuela en torno a la disciplina en la Facultad de Medicina de la Universidad de Antioquía en los años sesenta del sioglo XX, la cual logró impactar positivamente en otras áreas de la medicina y la formación de los primeros inmunólogos. En esta segunda parte, se relatan los hechos ocurridos posteriormente que llevaron a la consolidación de la inmunología como una de las disciplinas de mayor desarrollo científico en el departamento de Antioquia y, particularmente, en la Universidad de Antioquia.

\section{La Sección de Inmunología}

Regresé al país a principios de 1977 e inmediatamente asumí la dirección del Laboratorio de Inmunología, acéfalo desde el retiro de Marcos Restrepo. El impacto fue grande, debido a situaciones políticas del momento en la Facultad y en el Departamento de Microbiología y Parasitología, la actividad académica se reducía a participar en unos cursos de pregrado, además, el laboratorio tenía una infraestructura muy precaria. Sin embargo, asumí los retos que me había impuesto y los que la institución me requirió.

Administrativamente, se logró que el laboratorio se convirtiera en sección del Departamento de Microbiología y Parasitología, dándole una mayor autonomía y reconocimiento, pues, el anterior proyecto de tener una sección compartida con Medicina Interna se frustró con el retiro de Marcos Restrepo, aunque se continuó con la colaboración en alergología y reumatología.

El primer día de trabajo recibí la visita del nefrólogo Jaime Borrero y del cirujano Álvaro Velásquez, quienes dirigían el Grupo de Trasplantes de la Universidad de Antioquia y el Hospital San Vicente de Paúl. Ellos me invitaron a vincularme al grupo y realizar los estudios inmunológicos para trasplantes, básicamente, la tipificación HLA y el estudio de anticuerpos citotóxicos. Esta solicitud la consideré como un requerimiento institucional, pues el Programa de Trasplantes representaba el liderazgo de la Universidad de Antioquia en el país y América Latina en ese campo.

Orgullosamente, debo decir que fue el inicio de la inmunología de trasplantes en Colombia: la creación de un laboratorio que ha sido un referente en el país, la oportunidad de prestar un servicio clínico muy importante, vincularnos con la comunidad latinoamericana e internacional de inmunólogos de trasplantes e inmunogenetistas, nuestra participación en los talleres latinoamericanos de histocompatibilidad, iniciar una línea de investiogación que continúa siendo muy productiva, formar un número importante de inmunólogos con maestría y doctorado $y$, adicionalmente, tener una fuente de ingresos para el laboratorio que nos ha permitido una relativa solvencia económica. Esta labor se realizó con el apoyo de varias bacteriólogas, entre quienes destaco a Ángela María Arango, Margarita Correa, Betty Ángel, Beatriz Guzmán, Mabel Cristina Giraldo, Natalia García, Libia María Rodríguez, la bióloga Luz Marina Restrepo, el veterinario Juan Esteban Pérez y, principalmente, el microbiólogo Cristiam Mauricio Álvarez, que realizó, bajo mi dirección, su maestría y su doctorado y, al día de hoy, dirige el laboratorio de Inmunología de Trasplantes.

Paralelamente, a estas actividades en inmunología de trasplantes, se realizaron estudios de inmunogenética de poblaciones, principalmente autóctonas, dentro de los Talleres Latinoamericanos ya mencionados.

Como mencioné anteriormente, los programas de maestría en ciencias básicas creados en los años setenta turieron muy buena acogida en la ciudad y el país, pero por las condiciones políticas de esos años y el retiro de la Universidad de sus fundadores, algunos estudiantes estaban en una situación de incertidumbre para la realización de su trabajo de investiogación. Esta situación me permitió ayudar a quienes, además de ser antiguos amigos y compañeros, eran personas 
brillantes. Fernando Montoya, compañero en medicina y en la maestría, estudió las diferencias entre sexos en la respuesta inmune al Paracoccidiodes brasiliensis y, logramos demostrar, por medio de la inhibición de la migración de los macrófagos (MIF), que las hembras desarrollan una mejor respuesta inmune celular (1). Fernando hizo una destacada carrera académica en la Facultad de Medicina y fortaleció la alergología en la Universidad y la ciudad. Luis Rafael Caraballo, siendo estudiante de medicina en su nativa Cartagena, era un asiduo asistente a los cursos que se dictaban en el Departamento de Microbiología y Parasitología, luego de graduarse en la Universidad de Cartagena ingresó a la maestría en Microbiología. En su trabajo de investigación estudió los anticuerpos antilinfocíticos en pacientes con lupus eritematoso sistémico ${ }^{(2)}$. Luis regresó a la Universidad de Cartagena donde ha realizado una brillante carrera en investigación en alergias, reconocida nacional e internacionalmente. Con Luz Aristizábal, bacterióloga inteligente y dedicada, estudiamos el efecto de la estimulación con el mitógeno concanavalina A y antígenos micobacterianos en la formación de diferentes tipos de rosetas $\mathrm{E}$ en pacientes con tuberculosis ${ }^{(3,4)}$. Esta técnica fue la primera empleada para la identificación de las células T humanas ${ }^{(5)} y$, con algunas variaciones metodológicas, nos permitieron cuantificar y evaluar la respuesta funcional de los linfocitos T.

En el pregrado debo mencionar la vinculación al laboratorio del estudiante de medicina Augusto Ochoa, quien hacía parte del primer grupo de medicina al que me correspondió enseñarle. Augusto se entusiasmó con la inmunología y empezó a frecuentar el laboratorio y a participar en los estudios inmunológicos para trasplantes, convirtiéndose en el compañero de las frecuentes trasnochadas en que, contra el tiempo, teníamos que hacer estos estudios, pues aún no se disponía de los métodos de preservación del injerto y el donante. Una vez definida la muerte cerebral del donante, se mantenía con respiración artificial en condiciones clínicas muy inestables. Pude ayudarle a Augusto para que una vez graduado de médico se entrenara como inmunólogo en la Universidad de Minnesota. Augusto fijó su residencia en Estados Unidos donde ha realizado una brillante carrera como investigador en inmunología del cáncer. En el año 2015, la Universidad de Antioquia lo galardonó como egresado distinguido. Durante todos estos años hemos mantenido una amistad científica y personal muy cercana.

Otros hechos importantes fueron los cursos de inmunobiología dictados en el Departamento de Biología. Estos cursos me permitieron enseñar la inmunología más básica y utilizar como herramienta pedagógica el análisis de los experimentos más relevantes publicados sobre el respectivo tema. Además, fue la posibilidad de atraer estudiantes de biología a la inmunología, lo cual se logró exitosamente. La primera estudiante de biología que hizo su trabajo de grado bajo mi dirección fue Sara Claudia París, con quien profundicé en el estudio del fenómeno de la formación de rosetas E por los linfocitos T humanos; como resultado se originaron dos publicaciones internacionales, algo excepcional para un trabajo de pregrado en ese momento ${ }^{(6,7)}$.

Sara se vinculó al Departamento de Microbiología y Parasitología y, durante muchos años, fue mi más cercana colaboradora y el soporte del grupo en muchos aspectos técnicos y humanos de gran importancia. Otros biólogos que fueron sensibilizados por la inmunología y a quienes pude ayudar en sus tesis de maestría o doctorado son Luis Fernando Barrera y Mauricio Rojas, quienes han sido mis estudiantes, amigos y compañeros por muchos años. Esos cursos para biólogos continúan dictándose y son una fuente permanente de jóvenes brillantes y deseosos de hacer una vida en la ciencia.

Una actividad interesante que se realizó en la Sección de Inmunología durante esos años la hizo la dermatóloga Estela Prada de Castañeda, quien ensayó la inmunoterapia con dinitroclorobenceno (DNCB) para el tratamiento de las verrugas múltiples. Para ello, sensibilizaba a los pacientes mediante la aplicación tópica de DNCB en un área libre de verrugas y unas semanas más tarde lo aplicaba en las lesiones, las cuales se inflamaban y luego desaparecían por un "efecto espectador", consecuencia de la reacción de hipersensibilidad retardada inducida por el DNCB.

En 1977 asumió la decanatura de la Facultad de Medicina el profesor Federico Díaz, quien me solicitó preparar la propuesta para la creación del Centro de Investigaciones Médicas (CIM) que se logró en el 1980, cuando ya era decano Antonio Yepes, que 
me honró nombrándome como su primer director. En conversación con el decano Yepes, me consultó quién debería asumir la dirección de la Sección de Inmunología, a lo cual respondí que la pediatra Diana García de Olarte (Figura 1), era la persona indicada. Sin embargo, le agregué que su nombramiento le podría generar dificultades con el Departamento de Pediatría, dado su liderazgo académico en esa dependencia. Su respuesta fue inmediata y contundente: "es más fácil conseguir un pediatra que un inmunólogo”, la nombró jefe de la Sección de Inmunología.

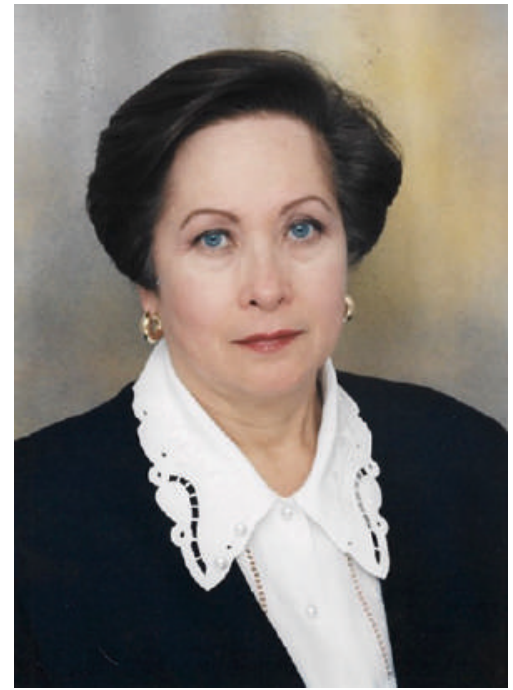

Figura 1. Diana García de Olarte, médica pediatra e inmunóloga clínica que dirigió la Sección de Inmunología entre 1980 y 1995. Coordinadora del programa de maestría en Inmunología ICFES-BID, fundadora del Grupo de Inmunodeficiencias Primarias. Fuente: fotografía gentilmente proporcionada por la profesora Diana García de Olarte

Diana, una mujer brillante, creativa y poseedora de una gran energía, asumió ese reto con gran entusiasmo y dedicación. Luego de entrenamientos cortos en las universidades Wake Forest, Harvard y Londres sobre la biología de los polimorfonucleares neutrófilos, implementó las metodologías para evaluar la fagocitosis y la quimiotaxis de estas células ${ }^{(8)}$. Con Diana compartimos el laboratorio y realizamos algunos proyectos conjuntos, entre los que resalto la determinación de los niveles séricos de inmunoglobulinas en población normal de Medellín, en este participó la bacterióloga Luz Helena Posada.

Un logro importante en la gestión de Diana fue conseguirle al pediatra Helí Salgado, su antiguo discípulo, un entrenamiento en inmunodeficiencias primarias en la Universidad de Harvard. Al regreso de Helí, ambos crearon un ambicioso programa para el estudio inmunológico y el manejo clínico de pacientes con el síndrome de infección recurrente, lo que les permitió reclutar un número importante de pacientes con inmunodeficiencias primarias $y$ darle origen al Grupo de Inmunodeficiencias Primarias (IDP). Diana también fue pionera en el estudio de los efectos de las sustancias sicoactivas sobre el sistema inmune. Como lo describiré más adelante, desempeñó un papel fundamental en la maestría en Inmunología. Después de su retiro de la Universidad en el año 2000, Diana se marginó de toda actividad académica, pero su legado permanece por medio de sus discípulos en el exitoso grupo IDP y en la fundación que lleva su nombre $y$ promueve el estudio $y$ manejo de pacientes con inmunodeficiencias primarias: Fundación Diana García de Olarte para Ias Inmunodeficiencias Primaria (FIP).

\section{El laboratorio CentraI de Investigaciones (LCI)}

Durante mi gestión como director del CIM propuse que el antiguo Laboratorio de Nutrición, ubicado en el primer piso del edificio de Bioquímica (hoy edificio Manuel Uribe Ángel), se convirtiera en un laboratorio al servicio de los clínicos que requirieran en sus proyectos procedimientos no disponibles en otros laboratorios de la Facultad o del Hospital San Vicente de Paúl. La propuesta fue aprobada por el Consejo de Facultad y, al retirarme de la dirección del CIM en 1984, el decano Jaime Borrero me pidió que lo dirigiera. Le puse de presente que si yo lo hacía el LCI pudiera convertirse en otro laboratorio de inmunología, argumento que no lo convenció, pero que rápidamente fue una realidad. El crecimiento en el número de proyectos y de estudiantes, de Diana y míos, hizo que las instalaciones de la Sección de Inmunología fueran insuficientes $y$, mis proyectos y estudiantes, se desplazaron a este nuevo laboratorio. Esos años del LCI fueron muy productivos por los proyectos que adelantamos, así como por el número y la calidad de los estudiantes que se formaron. En 1985 logramos el primer proyecto con financiación internacional por 
Ia OMS para estudiar clonas de linfocitos T de pacientes con tuberculosis. Este proyecto permitió adquirir los equipos necesarios para el estudio in vitro de las respuestas inmunes celulares y, al biólogo Luis Fernando Barrera, realizar su trabajo de maestría. En esa ubicación el LCI permaneció hasta 1994, cuando nos mudamos al antiguo recinto de la Academia de Medicina, en el segundo piso del edificio central de la Facultad de Medicina, el cual fue remodelado para permitirnos tener unas instalaciones más acordes con el trabajo que realizábamos. Para este momento Colciencias ya había lanzado su política de clasificar los grupos de investigación, al presentarnos a la primera convocatoria decidí darle al grupo el nombre de Grupo de Inmunología Celular e Inmunogenética (GICIG), que permanece hasta el momento.

Otro hecho importante, fue la realización de mi año sabático entre 1991 y 1992 en McGill University (Montreal, Canadá) que me permitió familiarizarme con métodos moleculares que, llevaron a mi regreso a la adquisición de los primeros equipos necesarios para hacer inmunología e inmunogenética moleculares. Además, gracias al programa de retorno de Colciencias, se vincularon mis antiguos estudiantes Luis Fernando Barrera (después de terminar su doctorado en McGill University), Luz Marina Restrepo (que adelantó su doctorado en la Université de París, Francia y luego creó el grupo de Ingeniería de Tejidos), y Blanca Lucía Ortiz (que realizó su doctorado en la Universidad Autónoma de Madrid).

EI GICIG desarrolló inicialmente las líneas de inmunología de trasplantes, inmunogenética de poblaciones $y$ respuesta inmune en tuberculosis $y$ posteriormente, la línea de Autoinmunidad, liderada por la reumatóloga Gloria Vásquez, quien hizo su doctorado bajo la tutoría de Luis Fernando Barrera, actualmente la coordinadora del GICIG.

\section{La Sección de Virología}

La Sección de Virología del Departamento de Microbiología y Parasitología fue creada por el profesor José Hernán López, allí se implementaron las técnicas serológicas usuales para el estudio de las infecciones virales. En esta sección se desarrolló una colaboración con la Universidad de Wisconsin para el estudio de zoonosis virales que permitió la formación de algunos médicos veterinarios de maestría en esa prestigiosa universidad norteamericana. Entre ellos estaba Jorge Eliecer Ossa, adscrito a la Facultad de Medicina Veterinaria, con quien hice una amistad cercana dado su interés por la inmunología. Jorge se retiró de la universidad para hacer su Ph.D. en el Virginia Polytechnic Institute, a su regreso se vinculó con la Universidad Nacional en Bogotá. Para esta época, 1983-84, se presentó una vacante en una plaza en la Sección de Virología y logramos convencerlo de regresar a Medellín y asumir su dirección.

Vinculado nuevamente, Joroge impulsó, básicamente, dos líneas de investigación: respuesta inmune al citomegalovirus e inmunología de la reproducción, que luego darían origen al Grupo de Inmunovirología, hoy dirigido por María Teresa Rugeles, quien ha realizado estudios muy importantes en inmunología del SIDA y, al Grupo de Reproducción, pionero en ese campo y dirigido exitosamente por Ángela Patricia Cadavid. Jorge desempeñó un papel importante en los desarrollos posteriores de la inmunología.

\section{La nueva maestría en Inmunología}

A mediados de los años 80 , el gobierno colombiano hizo un préstamo con el Banco Interamericano de Desarrollo (BID) para fortalecer las ciencias básicas en las universidades públicas y encomendó su dirección al ICFES, por lo que el programa se conoció como Programa ICFES-BID. Para seleccionar los programas se solicitaron propuestas a todas las universidades públicas y, Diana García, Jorge Ossa y yo presentamos la propuesta para un programa de maestría en Inmunología, esta fue seleccionada, junto con otras cuatro de la Universidad de Antioquia.

Este programa permitió la modernización de la infraestructura de los laboratorios, las pasantías internacionales de los profesores y las becas para los estudiantes matriculados. La coordinación del programa Maestría en Inmunología estuvo a cargo de Diana García hasta su finalización en 1993. La primera cohorte de estudiantes inició sus estudios en febrero de 1987, la conformaron el médico Ricardo Cardona y la bacterióloga Fabiola Toro, ambos bajo la dirección de Diana García, la médica Ángela Patricia Cadavid, bajo la tutoría de John Jairo Estrada y, el biólogo Luis Fernando Barrera a mi cargo, quienes se graduaron en mayo de 1989. Además de esta primera cohorte, otras posteriores permitieron formar un número 
considerable de inmunólogos, algunos se vincularon con la Universidad de Antioquia, otros regresaron a sus instituciones y algunos se establecieron en el exterior. Muchos de ellos completaron su formación a nivel doctoral. EI programa ICFES-BID terminó en 1993 y cumplió con creces su objetivo de fortalecer las ciencias básicas, en nuestro caso, la inmunología.

\section{El posgrado en Ciencias Básicas Biomédicas}

Al terminarse el Programa ICFES-BID, para Diana García, Jorge Ossa y mi persona era claro que la maestría en Inmunología debía continuar con el apoyo de la Universidad. También teníamos claro que durante esos años otros grupos, de áreas diferentes a la inmunología, se habían fortalecido y no tenían un programa de posgrado para continuar su desarrollo natural. Además, en ese momento, Colciencias estaba promoviendo la creación de programas de doctorado.

Después de discutirlo extensamente, invitamos a otros grupos de la Facultad, otras dependencias de la Universidad y a otras instituciones, como la Corporación para Investigaciones Biológicas (CIB) y el Centro Internacional para Educación e Investigación Médica (CIDEIM) de Cali, a presentar una propuesta conjunta para la creación de un programa marco de posgrado en Ciencias Básicas Biomédicas que incluyera maestría y doctorado. Así se hizo con el liderazgo de Jorge Ossa y la fisióloga Hilda Nora Jaramillo, quienes elaboraron la propuesta que fue aprobada por la Universidad y que luego sustentamos con Jorge Ossa ante los evaluadores nacionales e internacionales en Bogotá. El programa se inició en 1994 y su primer director fue Jorge Ossa. Posteriormente, con la expedición del Estatuto General de la Universidad de Antioquia en 1995, que contempla la existencia de Corporaciones Académicas conformadas por diferentes dependencias, Jorge lideró la conversión del programa de posgrado en la Corporación Académica de Ciencias Básicas Biomédicas, que continúa administrando las maestrías y los doctorados, incluyendo las que tienen énfasis en inmunología.

\section{La Inmunología en la Corporación para Investiga- ciones Biológicas (CIB)}

En 1970 un grupo de profesores de la Facultad de Medicina crearon la CIB con el objetivo de manejar recursos de investigación en una forma más ágil que la administración de la Universidad. Sin embargo, con la crisis política de mediados de los años setenta, muchos de ellos se retiraron de la Universidad y continuaron con la CIB como una institución privada. La CIB bajo el liderazogo de Ángela Restrepo y William Rojas, logró convertirse en una de las principales instituciones de investigación del país con gran reconocimiento internacional ${ }^{(9,10)}$.

Como mencioné anteriormente, Ángela Restrepo inició en la Facultad de Medicina su largo y fecundo proceso de investigación sobre la paracoccidioidomicosis, tarea que continúo en la CIB reuniendo un grupo de jóvenes talentosos y bien formados para estudiar diferentes aspectos de esta micosis, incluyendo la inmunología. Particularmente, Luz Helena Cano después de su maestría en Microbiología en la Universidad de Antioquia, hizo su doctorado en la Universidad de São Paulo (Brasil) y regresó a la CIB a investigar la inmunología de la paracoccidioidomicosis empleando el modelo murino, con resultados reconocidos internacionalmente ${ }^{(10)}$.

Fabiola Montoya de Restrepo después de su retiro de la Universidad se vinculó con la CIB y estableció un laboratorio de inmunogenética en el que hizo estudios de asociación de HLA con malaria y paracoccidioidomicosis $y$, de frecuencias de los polimorfismos de HLA en comunidades indígenas, además, participó en los talleres latinoamericanos de Histocompatibilidad. Posteriormente, el reumatólogo Juan Manuel Anaya realizó en la CIB estudios inmunogenéticos en artritis reumatoide y síndrome de Sjögren.

\section{El periodista de la inmunología}

En mis viajes por América Latina, uno de los comentarios más frecuentes de parte de muchos inmunólogos fue sobre el impacto positivo que turo en ellos el libro de inmunología del médico internista William Rojas (Figura 2).

En una entrevista que gentilmente me concedió el 18 de abril de 2018, me relató el origen y el posterior desempeño de su libro. La historia es la siguiente. En 1972 viajó a Londres para tomar los cursos preparatorios para su ingreso al Royal College of Physicians y, dentro de estos, tomó un módulo de inmunología. 
A su regreso al país, sus colegas de la Clínica Soma, donde tenía su consultorio, le solicitaron que les diera un curso de inmunología. Para este fin, William Rojas hizo sus propios dibujos e imprimió en esténcil un texto de inmunología clínica para distribuirlo entre Ios participantes (Figura 2). Además, acudió a su amigo Efraín Upegui, propietario de la Editorial Colina, para que le facilitara un dibujante para preparar las filminas de sus conferencias. Efectivamente, el señor Upegui le colaboró, pero además lo convenció de que estas conferencias se convirtieran en un libro, cuya primera edición se publicó en $1973^{(11)}$.

Posteriormente, se publicó por el Fondo Educativo Interamericano Wesley en México y por el Fondo de Editorial de la CIB. Un hecho importante fue que la CIB logró un convenio con la OPS para que sus libros fueran distribuidos en toda Latinoamérica, lo que permitió que este texto llegará a ser muy popular en todo el continente.
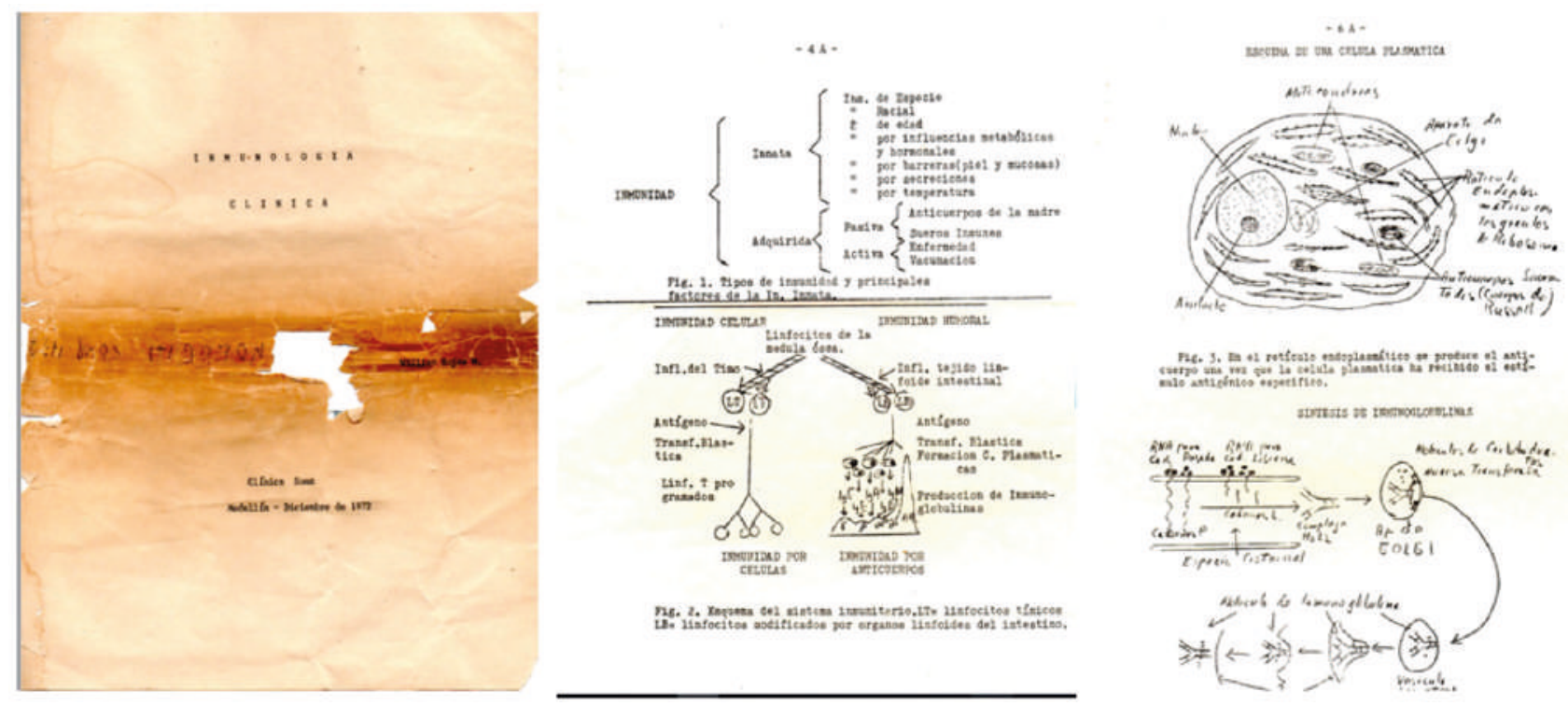

Figura 2. Texto original del libro Inmunología Clínica de William Rojas, impreso en esténcil en el 1972, con dibujos del autor. Fuente: documentos escaneados por Luis $\mathrm{F}$ García con autorización de William Rojas

Hasta el momento se han hecho 18 ediciones (Figura 3), cerca de 85.000 ejemplares en total. En las quince primeras ediciones fue un libro de un solo autor, pero en las tres últimas se convirtió en un libro en el que él pasó a ser editor principal y, los coeditores, Juan Manuel Anaya, Luis Miguel Gómez, Luz Elena Cano, Beatriz Aristizábal y Damaris Lopera. Curiosamente, William Rojas no ha sido un inmunólogo de carrera y se considera a sí mismo como un "periodista de la inmunología”, fascinado con el funcionamiento del sistema inmune y la investigación que se realiza en esta disciplina. Cuenta él que la información para mantener actualizado el libro durante casi cinco décadas proviene de un club de revistas con estudiantes de pregrado con quienes revisa las 20 principales revistas de inmunología para seleccionar los avances que considera más significativos e incluirlos en la edición siguiente. 


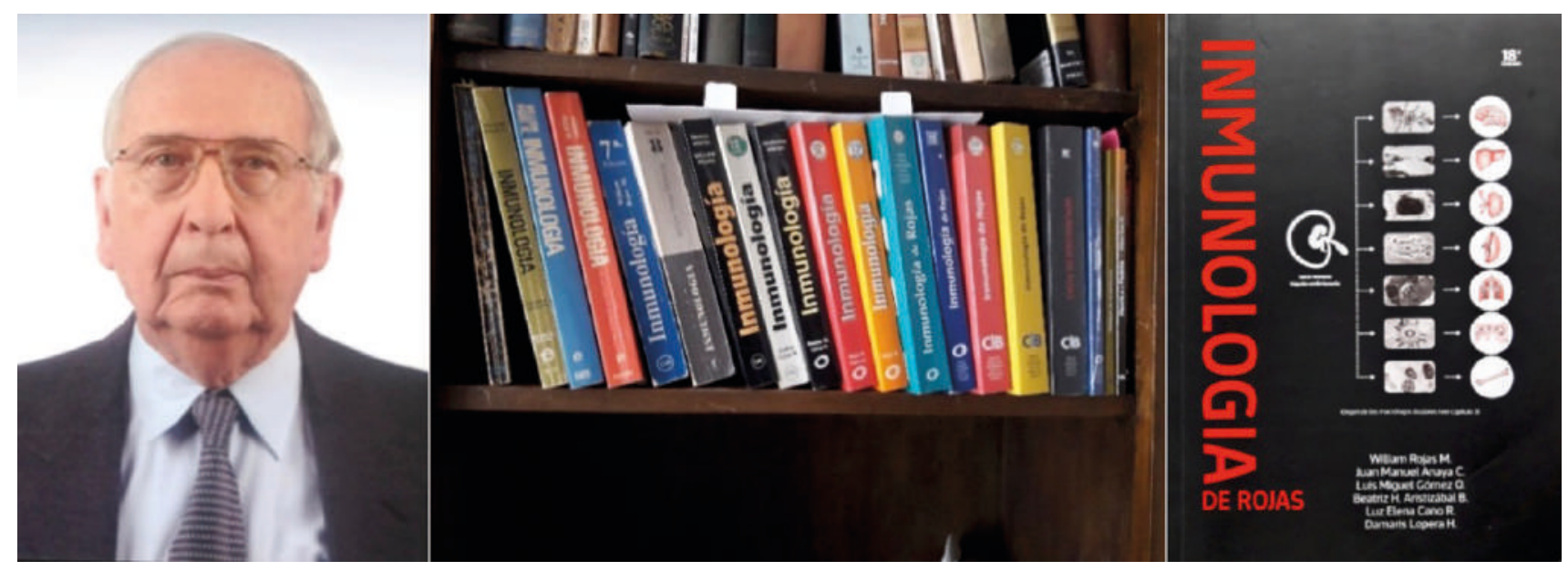

Figura 3. William Rojas, médico internista. Autor del libro de texto clásico Inmunología, con 18 ediciones y más de 85.000 ejemplares vendidos en América Latina. Fuente: Fotografías gentilmente proporcionadas por el Profesor William Rojas

\section{CONFLICTOS E INTERESES}

Ninguno por declarar.

\section{REFERENCIAS BIBLIOGRÁFICAS}

1. Montoya F, García-Moreno LF. Effect of sex on delayed hypersensitivity responses in experimental mouse paracoccidiodomycosis. J Reticuloend Soc. 1979;26:467-478.

2. Caraballo LR, Medina ME, García LF, Molina J. Lupus eritematoso sistémico: Poblaciones de linfocitos y anticuerpos linfocitotóxicos. Tribuna Médica. 1979;59:37-43.

3. Aristizabal L, García-Moreno LF. The effect of Concanavalin A on the formation of different types of E-rosettes. J Reticuloend Soc. 1981;29:117-125.

4. Aristizabal L, García LF. Formation of total and stable E-rosettes by lymphocytes from patients with pulmonary tuberculosis stimulated by Con A or PPD. J Clin Lab Immunol. 1985;16:31-36.

5. Jondal M. SRBC rosette formation as a human T lymphocyte marker. Scand J Immunol. 1976;5(suppl 5):69-76.
6. Paris SC, Garcia-Moreno LF. Formation of total, active and stable E-rosettes by human lymphocytes stimulated with Con A derivatives. J Clin Lab Immunol 1982;8:183-6.

7. Paris SC, García LF. Evidence that active and stable E-rosette forming cells belong to different human T lymphocyte subpopulations. Allergol Immunopathol. 1983;11:161-8.

8. García de OD, Toro F. Quimiotaxis bajo agarosa. Método para medir la quimiotaxis de los polimorfonucleares neutrófilos. Acta Med Col. 1986;11:52-6

9. Jaramillo Salazar H, Gómez Buendía H. 37 modos de hacer ciencia en América Latina. Bogotá: Colciencias; 1997.

10. Restrepo Moreno A, Rojas Montoya W, Sierra Botero DM, González Duque LM, Gómez Restrepo AJ, Gómez JC, et al. La CIB un lugar para el conocimiento 19702010. Medellín: Corporación para Investigaciones Biológicas; 2010.

11. Rojas W. Inmunología. Medellín: Editorial Colina; 1973. 